\title{
Pitfalls in the ultrasonographic diagnosis of gallbladder diseases
}

\author{
E.J. Fitzgerald ${ }^{1}$ and A. Toi ${ }^{2 *}$ \\ ${ }^{1}$ Department of Diagnostic Radiology, University Hospital of Wales, Heath Park, Cardiff CF4 3XW, UK, \\ and ${ }^{2}$ McMaster University Medical Centre, Hamilton, Ontario, Canada.
}

\begin{abstract}
Summary: Ultrasonography is rapidly replacing radiological techniques of gallbladder investigation. While ultrasonography is highly accurate, there are technical, anatomical and diagnostic pitfalls which will trap the unwary. This presentation highlights the pitfalls which we have encountered, reviews the literature in this area and suggests techniques whereby these pitfalls may be avoided.
\end{abstract}

\section{Introduction}

Ultrasonography is now considered by many to be the method of choice of screening for gallbladder disease. The accuracy of this technique is claimed to exceed $90 \%{ }^{1,2,3}$ However, high false positive rates of $7 \%$ and false negative rates of $15 \%$ are still reported in some series. ${ }^{4,5}$ Since the diagnosis of gallstones by ultrasonography is often followed by cholecystectomy without further confirmatory studies, the avoidance, especially of false positive diagnosis is important. If false positive gallbladder diagnosis were to occur at the $1.5 \%$ rate reported by Allen-Mersh, ${ }^{5}$ then about 2,000 unnecessary cholecystectomies could occur in England and Wales alone, and 7,000 in the United States.

We have not been able to find any article listing the myriad of problems encountered at ultrasonographic investigation of the gallbladder and as a result we present the pitfalls and problems which we have experienced over the past eight years at our institutions and found quoted in other publications, and suggest methods to overcome these problems.

\section{Discussion}

The types of problems may be broken down roughly into three major groups: technical, anatomical and diagnostic. Technical problems relate to choice and

Correspondence: $\quad$ E.J. $\quad$ Fitzgerald, M.B., $\quad$ M.R.C.P.I., F.R.C.R.

* Present address: Toronto General Hospital, 200 Elizabeth Street, Toronto, Ontario, Canada M5G 2C4

Accepted: 16 December 1986
Table I Pitfalls in the ultrasonographic diagnosis of gallbladder disease

Technical:

1. Mis-identification: bowel for gall stone ligamentum teres hepatic granulomas aorta

2. Wrong technique: transducer, gain patient not moved

3. Pseudosludge and calculi due to side lobe artefact.

4. Communication: typographical and documentation errors

Anatomical and physiological variants

1. Gallbladder folds junctional fold valves of Heister narrow gallbladder folded on itself

2. Gallbladder fold mimics dilated bile duct.

3. Sludge filled gallbladder looks like liver tissue.

4. Agenesis.

5. Duct stones with agenesis

6. Gallbladder ectopia

7. After cholecystoenterostomy, food and gas mimic stones.

8. Fundal and Hartmann stones not noticed.

Diagnostic errors:

1. Bouveret's syndrome called stone filled gallbladder.

2. Cholecystoses (adenomyosis and cholesterolosis).

3. Intramural gas called stones or cholecystosis.

4. Porcelain gallbladder.

5. Milk of calcium bile.

6. Sludge called tumour, polyp or stone and vice versa.

7. Gallbladder thickening called cholecystitis.

8. Post-cholecystectomy scar and clips called disease.

9. Food and gas called stones after cholecystoenterostomy.

10. Asymptomatic gallstones taken as cause of symptoms.

11. Cancer not recognized or considered. 
use of equipment and performance of the ultrasonographic examination. Anatomical problems relate to normal anatomical variants as well as physiological changes in the gallbladder and bile which may be misinterpreted as disease. Diagnostic problems relate to errors of interpretation of ultrasonographic appearances.

\section{Technical problems}

For elective studies, the patient should be fasted for 12 hours to allow adequate gallbladder distension to aid the examination and to prevent mistaking as abnormal, the normally thickened wall of the physiologically contracted gallbladder. ${ }^{6}$

Incorrect identification of another organ (bowel, stomach, duodenum, aorta, ligamentus teres) ${ }^{7}$ as the gallbladder will cause errors. Only the meticulous identification of landmarks such as the right portal vein and following the main lobar fissure to the gallbladder can avoid such errors. We have encountered a case of an ovarian dermoid on a long pedicle migrating to the subhepatic fossa adjacent to the gallbladder. Torsion of the pedicle led to pain and tenderness typical of gallbladder disease. Ultrasonographically, this mass mimicked cholecystitis, with 'stones' and debris which shadowed and moved with alteration of patient position.

Once the gallbladder has been located, the examiner must choose transducers with a suitable focal range. The common transducers used for abdominal examination (3.5 MHz long focus) of ten give poor, noise filled images of the gallbladder which may lie only $2-3 \mathrm{~cm}$ from the skin. Such gallbladders are adequately examined only with smaller crystal transducers of higher frequency (e.g. $5 \mathrm{MHz}$ ) having a focal zone at the gallbladder depth. We do not hesitate to employ high frequency linear array scanners if the gallbladder is very superficial and obscured in the main beam noise zone of the sector equipment. Alternatively with such superficial gallbladders, a standoff device may be used or the liver itself may be employed as a standoff to place the gallbladder within the focal zone of the transducer.

The absence of a distal acoustic shadow reduces the probability of an echo being a gallstone from $100 \%$ to $61 \% .^{10}$ All gallstones should cast shadows irrespective of size or composition. " The demonstration of such shadows is entirely dependent on an appropriately narrow beam profile, as determined by transducer selection and machine settings. If the calculus can stop enough of the beam to prevent sound transmission beyond it, shadowing will occur (Figure 1).

The characteristics of the 'shadow' have also been utilized. A calculus' shadow is said to be 'clean' and sharply defined. This is contrasted to the indistinctly marginated and reverberation echo filled 'shadow'

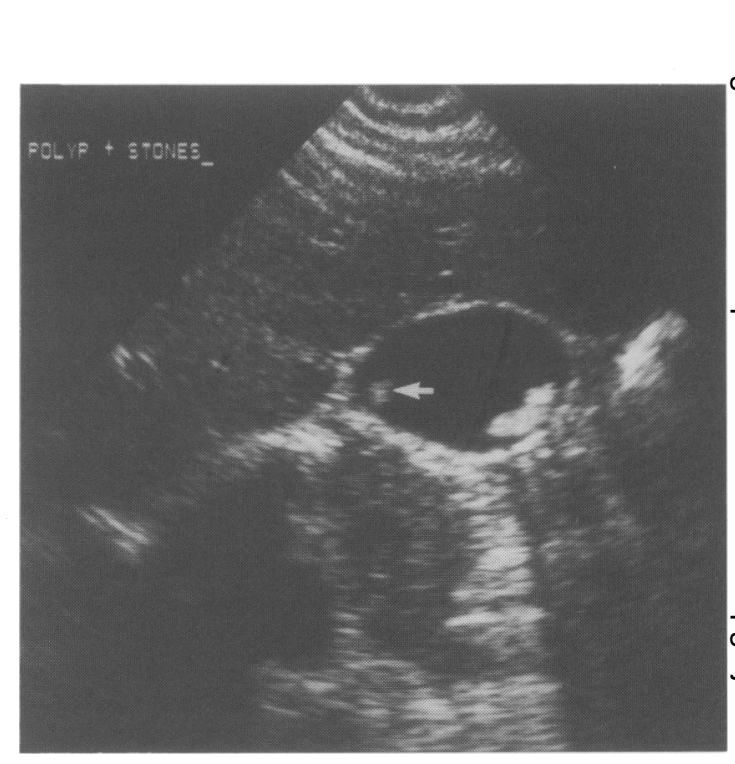

Figure 1 Stones and polyp (arrow) in the gallbladder. Note the clear shadowing behind the calculi and the absence of shadow behind the polyp.

associated with intestinal gas. ${ }^{12,13}$ We have found this to be a difficult sign to employ with confidence.

Failure to move the patient during the examinatiog is another source of error. Gallstones are diagnosed. most confidently if they can be shown to move durifis the examination by having the patient assume $\vec{a}$ decubitus or erect position. ${ }^{10}$ We have all too frequently seen stones for the first time when they moved as the patient changed position (Figures $2 a$ and $b$ ).

Artifactual side lobe echoes from adjacent structures are frequently written into the gallbladder lumen where they can be mistaken for sludge or calculi. ${ }^{14}$ Such spurious echoes are not constant as the transducer or patient are moved. The operator needs to be familiar with the artifactual echoes peculiar to his/her equipment.

There are some patients whose habitus will defeat the most skilled examiner. In such cases we recommend further gallbladder evaluation with alternative imaging techniques.

\section{Anatomical and physiological variants}

Several normal anatomical structures and variants in, and about, the gallbladder have been mistaken for $\underset{\mathrm{E}}{ }$ disease. Similarly, varying normal physiological states may create problems.

Folds are frequently present in the gallbladder. They cause confusing echoes and they may cast $\stackrel{\oplus}{+}$ shadows. ${ }^{15}$ The junctional fold at the junction of the $T$ body and infundibulum of the gallbladder and the $\overline{0}$ folds of the valves of Heister are a common source of $\Omega$ 

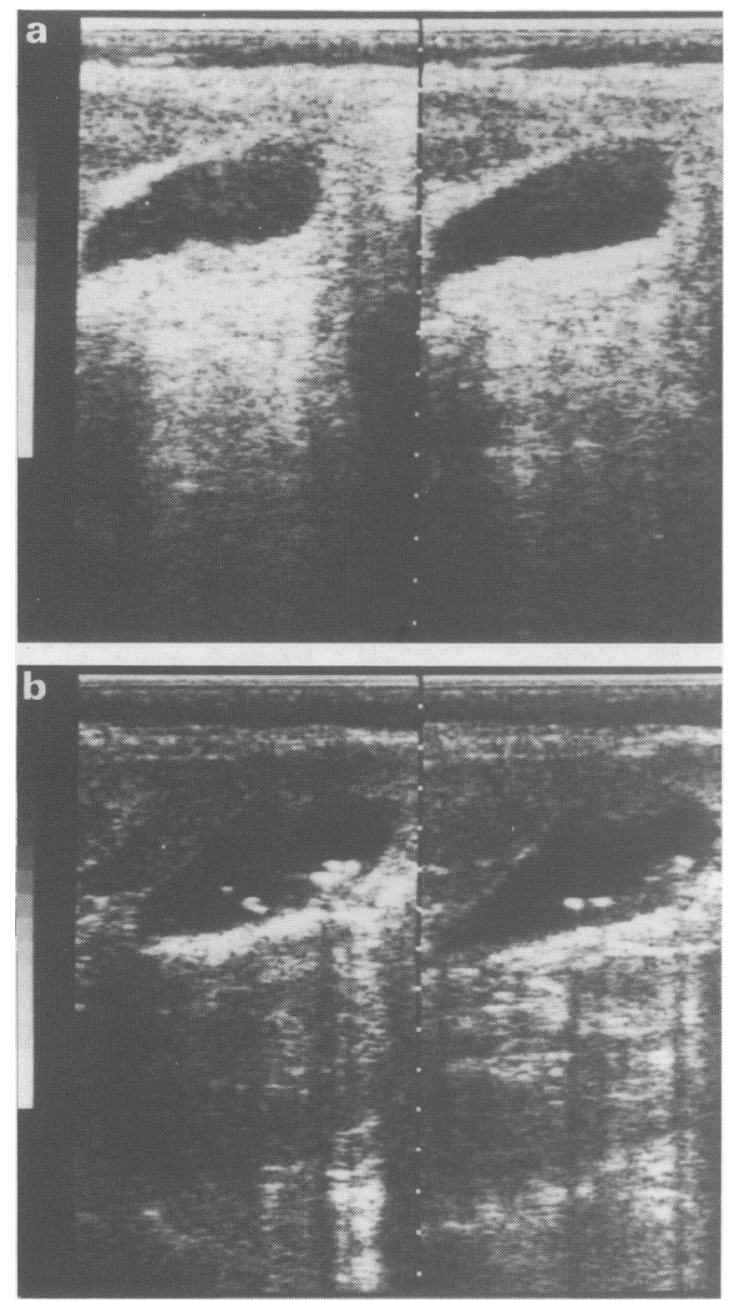

Figure 2 (a) The multiple gallstones layered on the dependent gallbladder wall are difficult to detect as machine gains are too high and transducer focus is too deep. (b) the calculi become easily visible when the patient is moved and the stones float free. Also, gains have now been set appropriately.

confusion. It has been suggested that if shadowing is seen from the region of the neck of the gallbladder in the absence of dilation of the organ, then one should regard the shadowing with suspicion. ${ }^{16}$ Such shadowing may result from sound attenuation and reflection by folds or from refractive effects seen in any area where fluid and solid interfaces coexist ${ }^{17}$ (Figure 3).

Similarly, a long narrow gallbladder folded back on itself may produce a 'septum' parallel to the long axis of the gallbladder. This may produce echoes which have been mistaken for stones. ${ }^{18} \mathrm{~A}$ similar redundant

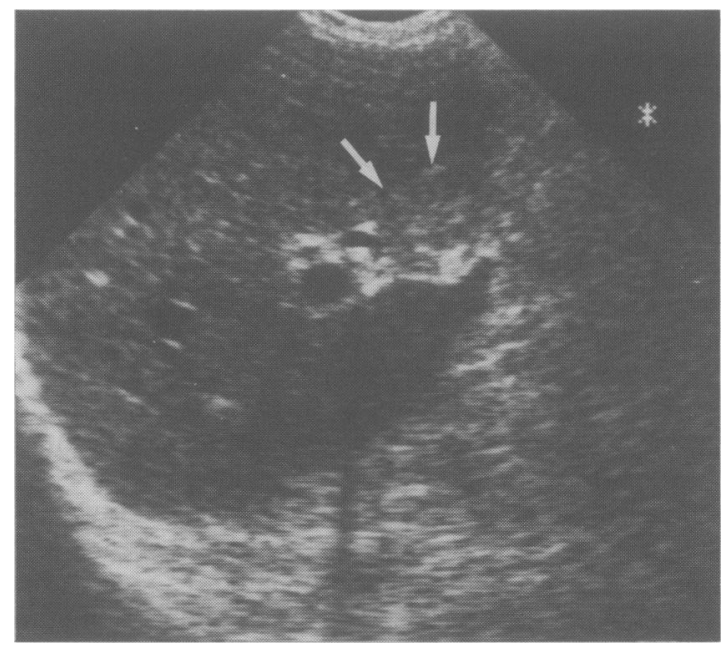

Figure 3 A kink in the hepatic artery near the neck of the gallbladder creates a strong specular echo and shadow which mimic a calculus in the gallbladder neck. Views in other directions and Doppler assessment revealed its true identity. Note also that the adjacent gallbladder is filled with sludge and virtually isoechoic with the liver. It is recognized only through identification of the subtle gallbladder-liver interface line (arrows).

or folded neck of gallbladder may mimic a dilated common bile duct. ${ }^{19}$

Failure to demonstrate a physiologically distended gallbladder in a fasting patient is reported to indicate a diseased gallbladder ${ }^{10,12}$ In our experience, it is very rare not to be able to locate an even minute, contracted, diseased gallbladder if correct transducers and techniques are employed. We have, however, encountered gallbladders which are distended with echogenic bile that is iso-echoic with liver. This renders the gallbladder invisible until the specular echo of its wall becomes visible or a small pocket of non-echogenic bile is identified (Figure 3). Gallbladder agenesis is an uncommon condition occurring in only $1 / 2500$ to $1 / 5000$ patients..$^{20}$ It is a diagnosis of exclusion which should be entertained only if the gallbladder fossa is clearly visible and shown not to contain the gallbladder and all sites of gallbladder ectopia have been assessed. These include the abdominal wall, beneath the left lobe of the liver or retroperitoneally. ${ }^{21}$ While gallbladder agenesis is rare, it does not result in immunity from cholelithiasis, since gallstones may form in the bile ducts of patients lacking a gallbladder. ${ }^{20}$

Food ${ }^{22}$ or air, ${ }^{23}$ may enter the gallbladder and bile ducts after surgical or spontaneous connections of either the gallbladder or bile ducts to bowel. Such echoes can be mistaken for calculi if the appropriate history is not available. A radiograph of the gallblad- 
der area may confirm the presence of gas, but in our experience, ultrasonography is more sensitive in detecting such small gas collections. In such cases, the examiner may be able to differentiate the less distinct echoes arising from gas as opposed to calculi ${ }^{13}$ to reach the correct diagnosis (Figure 4).

The specific gravity of bile may exceed that of gallstones and cause them to float. Stones may thus be overlooked as they lie in the reverberation echo filled non-dependent part of the gallbladder. Recognition of the characteristic shadow may be the only clue which leads to demonstration of such calculi. Bile specific gravity is increased by oral cholecystographic agents ${ }^{24}$ and floating stones should be considered if such studies have been recently performed. However, Carroll ${ }^{25}$ demonstrated that floating could occur without prior contrast administration (Figures $5 \mathrm{a}$ and $5 \mathrm{~b}$ ). Stones which contain gas, ${ }^{26}$ gas and calcium ${ }^{27}$ and pure cholesterol stones ${ }^{22}$ float in normal bile. As gascontaining calculi also float, this sign cannot be used as an indication for chemotherapeutic treatment, despite the recommendation to do this. ${ }^{28}$

The fundus and neck of the gallbladder are suboptimally examined by ultrasound. We have missed even large calculi of several centimetres impacted in both the fundus and in Hartmann's pouch. In the fundus, such stones are generally impacted behind a 'waist' in the gallbladder, possibly due to adenomyomatosis. Because the fluid bile pocket appears to close toward the fundus, one tends to overlook the stone in the nonbile-filled fundal pouch which has the appearance of adjacent colon. It is likely that at ultrasonography, we commonly miss the small fundal thickened patch of adenomyomatosis which was easily recognized at oral cholecystography by the characteristic RokintanskyAschoff sinuses (Figures 6a and b). Similarly, in a steeply angled gallbladder, stones in Hartmann's pouch can be difficult to discriminate from the normal

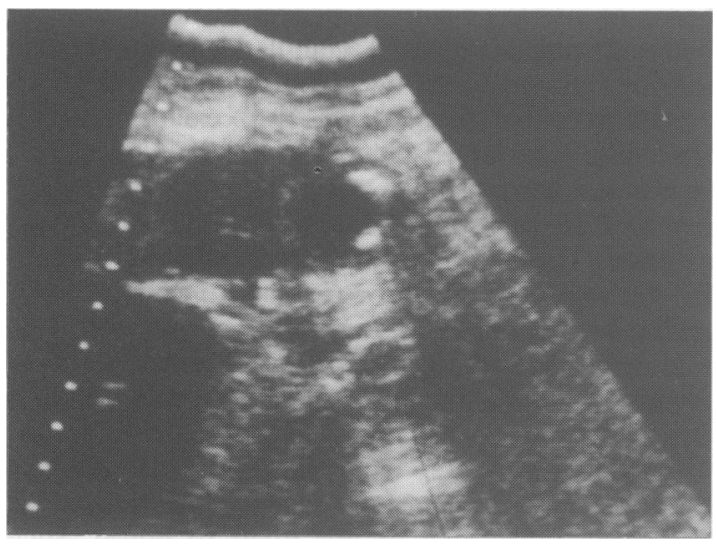

Figure 4 Air bubbles with emphysematous cholecystitis mimic floating stones.
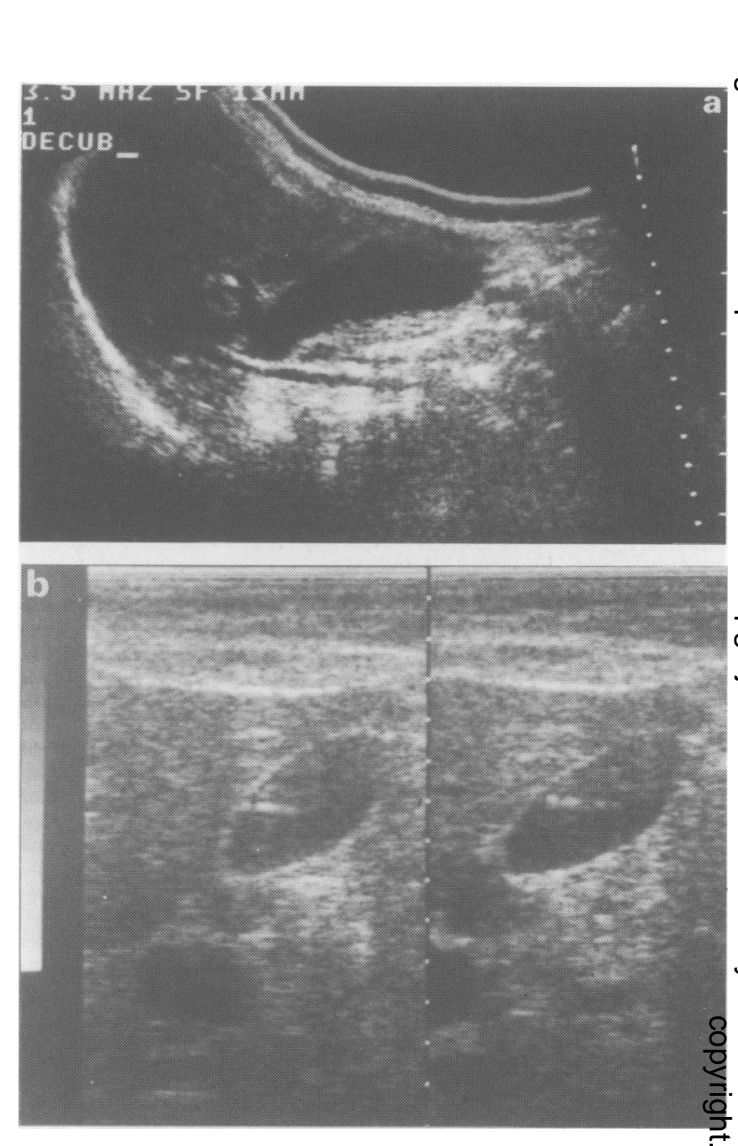

Figure 5 (a) Floating calculi in patient not associated with oral cholecystographic opacification. (b) Reverberation echoes mimic floating gallstones.

echogenic valves of Heister, portal structures and adjacent duodenum. Also, the gallbladder may not always be totally included in a single axial ultrasound image. The entire organ must be scanned meticulously in at least two planes to ensure that all parts have been seen, so that such errors may be obviated.

\section{Diagnostic errors}

Thickening of the gallbladder wall to $3 \mathrm{~mm}$ or more has been shown to be a non-specific sign which may be present in many disorders of the gallbladder, as a response of the normal gallbladder to disease elsewhere (e.g. hypoproteinaemia, heart failure) and even in the normal patient with physiological contraction of the gallbladder ${ }^{29}$ It should not be taken as a definitive sign of cholecystitis in the absence of other corroborating signs. Acute cholecystitis is not always associated with ultrasonographically detectable thickening of the gallbladder wall. This should be remembered in the sick, debilitated or post-operative patient in whom acalculous cholecystitis is sought. Wall thickening 


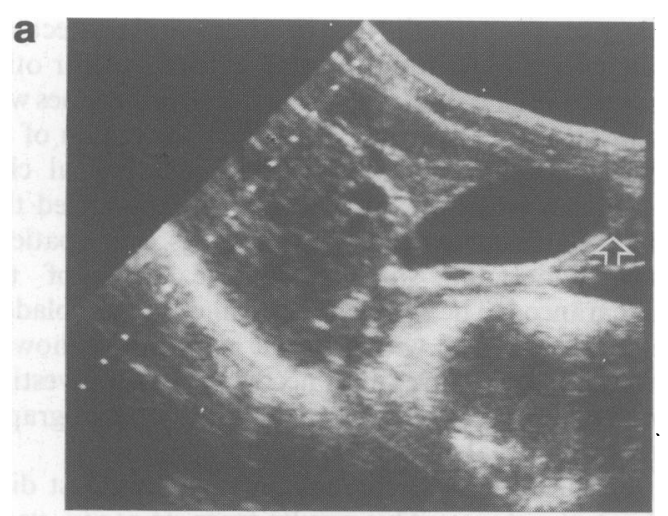

b

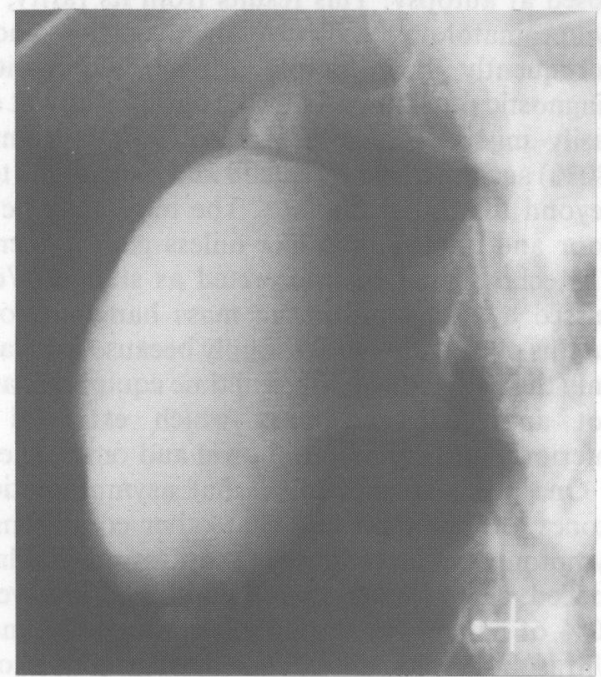

Figure 6 (a) Subtle thickening at the gallbladder fundus (arrow). This area of the gallbladder is frequently poorly assessed at ultrasonography. (b) Fundal adenomyomatosis shown by oral cholecystography.

may be detected in only about $2 / 3$ patients with this condition. ${ }^{30}$

Bile is rendered echogenic by the presence of calcium bilirubinate and cholesterol crystals. ${ }^{31}$ Such echogenic bile or sludge is commonly seen in the gallbladder of debilitated, fasting or intravenously fed patients. The sludge can be quite viscid and movement under gravity may be very slow or absent. If no movement is demonstrated then the picture may mimic a sessile gallbladder tumour. ${ }^{32} \mathrm{~A}$ repeat examination after regular meals have been resumed will allow the correct diagnosis. The presence of sludge does not exclude calculi which can be detected as echogenic, shadowing foci in the sludge. Simple sludge is generally homogenous and of uniform echogenicity. On occasion, sludge may coalesce as 'sludge balls' or 'tumefactive sludge'. These should not be mistaken for

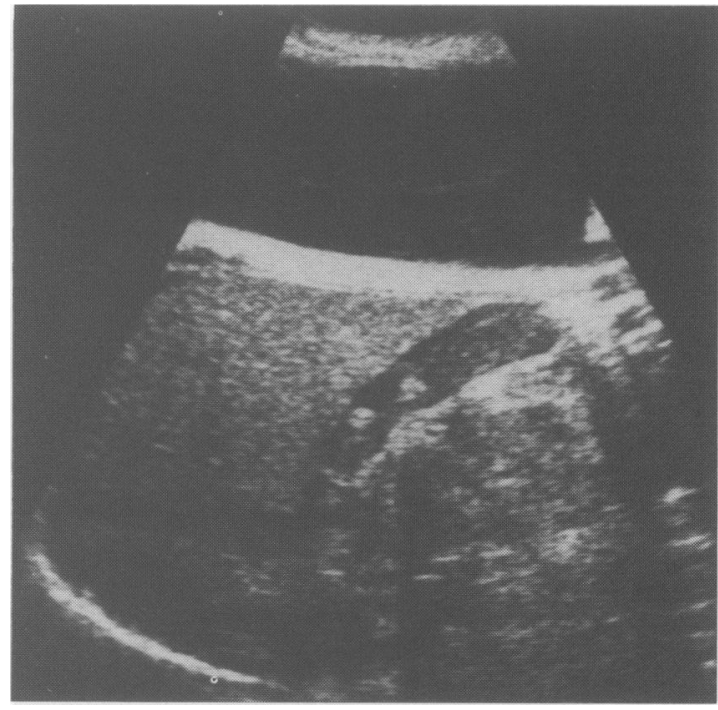

Figure 7 Patient on intravenous feeds whose gallbladder contains a sludge case and echogenic 'sludge balls' which do not shadow. These cleared spontaneously after return to normal diet. The visible shadow results from gas in the duodenum and should not be misinterpreted to arise from the contents of the gallbladder.

polyps or stones (Figure 7). After gallbladder stimulation, they should disappear.

The wall-echo-shadow (WES) triad ${ }^{33}$ or double-arcshadow $^{34}$ has been felt to be characteristic for gallstones within a contracted gallbladder. Recently a case of Bouveret's syndrome or gallstone ileus mimicking this sign has been reported..$^{35}$ The falsely positive WES sign resulted from imaging the perforated gallstone inside bowel lumen. This is an extremely rare condition but it should be considered especially in women aged over sixty who complain of upper intestinal obstruction in addition to gallbladder symptoms.

Several other disorders can give rise to appearances similar to the WES triad, namely porcelain gallbladder (Figures $8 \mathrm{a}$ and $\mathrm{b}$ ), ${ }^{36}$ emphysematous gallbladder, milk of calcium bile ${ }^{37}$ or gallbladder wall microabscesses. ${ }^{38}$ Thus careful attention should be given to clearly identifying the features of the WES triad so that these other potentially more clinically significant conditions will not be overlooked. If this triad cannot be demonstrated with confidence, then a plain film of the abdomen or computed tomography may be necessary. If porcelain gallbladder is suspected, then further investigation of both the gallbladder for malignancy and the liver for metastases is indicated since there is a $22 \%$ incidence of gallbladder malignancy in this condition. ${ }^{39}$

When echoes and shadows without definite evidence of a gallbladder wall are seen, careful atten- 

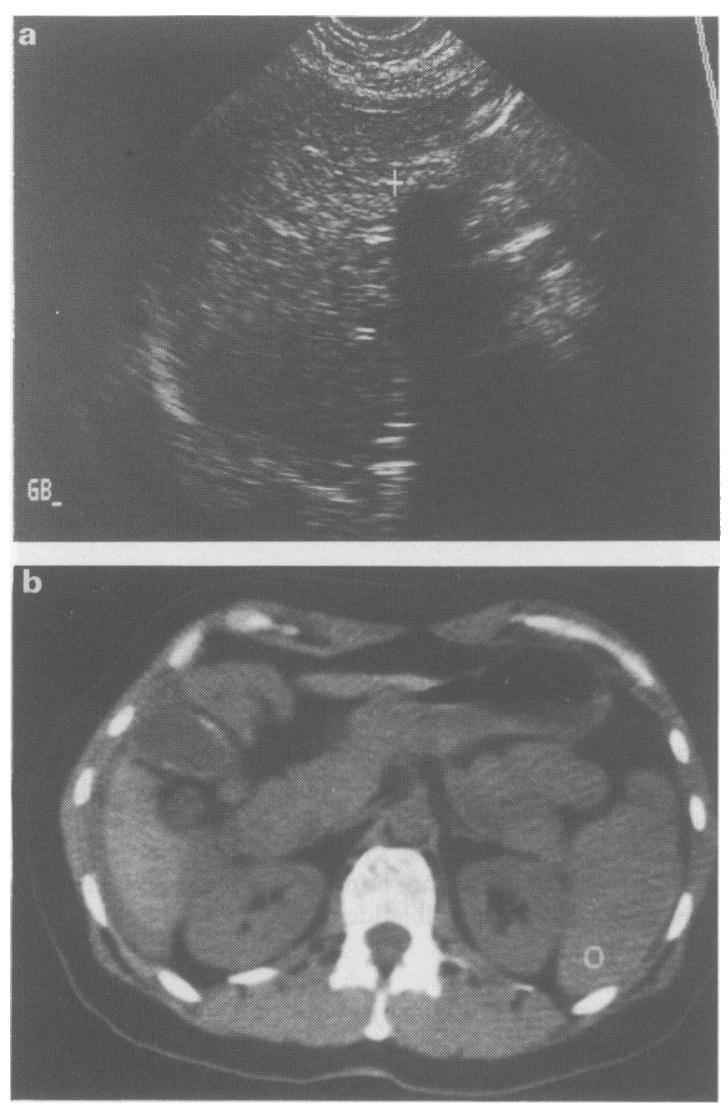

Figure 8 (a) Stone-like shadow in the gallbladder fossa suggesting the wall-echo-shadow complex of cholelithiasis. (b) Computed tomographic scan reveals that the shadowing results from mural calcification in this patient with porcelain gallbladder who has no calculi.

tion must be paid to the site of origin of the echoes. Adenomyomatosis with stones in the gallbladder wall can give this appearance. Intramural gas may give a similar appearance, but the presence of typical gas reverberation behind the echoes may help to differentiate this from calculi. ${ }^{40}$ However, in the presence of a fluid-filled gallbladder such intramural gas may be confused with gas in the bowel. In the case of intramural gas, a shift in position of the patient produces a gravity-induced equivalent shift of intramural and intraluminal gas, thus helping to confirm the diagnosis. ${ }^{41}$ Indeed, a definitive diagnosis of emphysematous cholecystitis cannot be made without the aid of radiological studies. ${ }^{41}$

The post-cholecystectomy gallbladder fossa can present confusing appearances. A history of cholecystectomy may not be elicited in patients who may have forgotten the procedure or in whom cholecystectomy was incidental to surgery being carried out for other reasons. After gallbladder removal, strong echoes with $\frac{3}{8}$ acoustic shadowing may arise from the region of the $\stackrel{\curvearrowright}{\circ}$ gallbladder bed. ${ }^{42}$ In the absence of surgical clips $\subseteq$ which can cause this appearance ${ }^{43}$ it is assumed that $\overrightarrow{\vec{A}}$ scar collagen gives rise to these findings. Some patients have undergone surgery on the basis of this appearance. ${ }^{42}$ The surgical removal of the gallbladder $\overline{\bar{m}}$ may be confirmed with a simple radiograph showing $\overparen{\mathbb{D}}$ the presence of clips, but if necessary, other investiga- $\frac{\Omega}{\circ}$ tions such as endoscopic retrograde cholangiography $\approx$ can be undertaken.

Gallbladder cancer is not uncommonly first diag- $\overrightarrow{.}$ nosed at autopsy. This results from its rarity, vague $\tilde{\sigma}$ symptomatology, difficulty of diagnosis and, not $\bar{D}$ infrequently, from simply not considering it as a 3 diagnostic possibility. Ultrasonographically, it can be easily missed for several reasons. It is commonly $\omega$

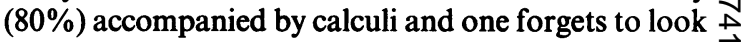
beyond the initial findings. The mass may be echo- $\vec{r}$ poor and appear fluid-like unless gain is increased. N The echoes may be interpreted as sludge. We have $ᄋ$ missed a $10 \mathrm{~cm}$ infiltrating mass hanging from the fundus of the gallbladder simply because we examined $\subsetneq$ only the gallbladder with real time equipment and did not appreciate the mass which extended more $\vec{\varphi}$ inferiorly and mixed into bowel and omental echoes

One needs to remember that asymptomatic gal stones are common and that other conditions hat symptoms similar to those of the diseased gallbladder., Indeed, acute cholecystitis is confirmed in fewer than $50 \%$ of patients in whom it is clinically diagnosed. ${ }^{45} \stackrel{\circ}{\perp}$ Ultrasonography, unlike cholecystography, allows the entire right upper quadrant to be evaluated for $\underline{0}$ diseases in other organs which may be the source of the patient's symptoms. We stress that a request for ultrasonographic gallbladder assessment really entails examination of the entire upper abdomen and should not be confined to the gallbladder.

\section{Conclusions}

Ultrasonography provides a rapid and accurate method of evaluating the gallbladder and right upper $\frac{7}{0}$ quadrant for disease. As experience with the technique grows, we are becoming aware of pitfalls which will $\stackrel{N}{\sigma}$ trap the unwary and give rise to both false positive and $N$ false negative studies. Sound anatomical knowledge, స్ట adherence to optimal technique, strict diagnostic 0 criteria and knowledge of the pitfalls are necessary to avoid diagnostic inaccuracy and potential patient harm. One should not hesitate to use other diagnostic $\stackrel{\oplus}{+}$ modalities when there is diagnostic uncertainty or if 0 confirmation of the findings is important. 
1. Cooperberg, P.L. \& Burhenne, H.J. Real-time ultrasonography. Diagnostic technique of choice in calculus gallbladder disease. N Engl J Med 1980, 302: 1277-1279.

2. Raptopoulos, V.D., Moss, L., Reuter, K. \& Kleinman, $P$. Comparison of real-time and gray scale static ultrasonic cholecystography. Radiology 1981, 140: 153154.

3. De Lacey, G., Gajjar, B., Twomey, B., Levi, J. \& Cox, A.G. Should cholecystography or ultrasound be the primary investigation for gallbladder disease? Lancet 1984, i: 205-207.

4. Mattson, M.W., Sterchi, J.M. \& Myers, R.T. Accuracy of ultrasonography and oral cholecystography in the diagnosis of cholelithiasis. Am Surg 1981, 47: 80-81.

5. Allen-Mersh, T.G., Motson, R.W. \& Hatley, W. Does it matter who does ultrasound examination of the gallbladder? Br Med J 1985, 291: 389-390.

6. Leopold, G.R. Biliary ultrasonography. In Benk, R.N. (ed) Radiology of the Gallbladder and Bile Ducts. Saunders \& Co., Philidelphia, 1983, p. 211.

7. Cooperberg, P.L., Pon, M.S., Wong, P., Stoller, J.L. \& Burhenne, H.J. Real-time high resolution ultrasound in the detection of biliary calculi. Radiology 1979, 131: 789790.

8. Lee, J.K.T., Melson, G., Koehler, R.E. \& Stanley, R.J. Cholecystosonography: accuracy, pitfalls and unusual findings. Am J Surg 1980, 139: 223-228.

9. Callen, P.W., Filly, R.A. Ultrasonography localization of the gallbladder. Radiology 1978, 133: 687-691.

10. Crade, M., Taylor, K.J.W., Rosenfield, A.T., de Graaff, C.S. \& Minihan, P. Surgical and pathologic correlation of cholecystosonography and cholecystography. AJR 1978, 131: 227-229.

11. Filly, R.A., Moss, A.A. \& Way, L.W. In vitro investigation of gallstone shadowing with ultrasound tomography. J Clin Ultrasound 1979, 7, 255-262.

12. Sommer, F.G., Taylor, K.J.W. Differentiation of acoustic shadowing due to calculi and gas collections. Radiology 1980, 135: 399-403.

13. Suramo, I., Paivansalo, M. \& Vuoria, P. Shadowing and reverberation artifacts in abdominal ultrasonography. Eur J Radiol 1985, 5: 147-151.

14. Fiske, C.E. \& Filly, R.A. Pseudosludge: a spurious ultrasound appearance within the gallbladder. Radiology 1982, 144: 631-632.

15. Sukov, R.J., Sample, W.F., Sarti, D.A. \& Whitcomb, M.J. Cholecystosonography - the junctional fold. Radiology 1981, 133: 435-436.

16. Kappelman, N.B. \& Sanders, R.C. Ultrasound in the investigation of gallbladder disease. $J A M A$ 1978, 239: 1426-1428.

17. Sommer, F., Filly, R.A. \& Minton, M.J. Acoustic shadowing due to refractive and reflective effects. AJR 1979, 132: 973-977.

18. Arnon, S. \& Rosenquist, C.J. Gray scale cholecystosonography: an evaluation of accuracy. AJR 1976, 127: 817818.

19. Laing, F.C. \& Jeffrey, R.B. The pseudo-dilated common bile duct: ultrasonographic appearance created by the gallbladder neck. Radiology 1980, 135: 405-407.

20. Mouzas, G. \& Wilson, A.K. Congenital absence of the gallbladder with stone in the common bile duct. Lancet 1953, i: $628-629$.

21. Vanderpool, D., Klingensmith, W. \& Oles, P. Congenital absence of the gallbladder. Am Surg 1964, 30: 324-330.

22. Gooding, G.A.W. Food particles in the gallbladder mimic cholelithiasis in a patient with cholecystojejunostomy. J Clin Ultrasound 1981, 9: 346-347.

23. Simeone, J.F., Mueller, P.R., Ferruci, J.T., Harbin, W.P. \& Wittenberg, J. Significance of nonshadowing focal opacities at cholecystosonography. Radiology 1980, 137: 181-185.

24. Scheske, G.A., Cooperberg, P.L., Cohen, M.M. \& Burhenne, H.J. Floating gallstones: the role of contrast material. J Clin Ultrasound 1980, 8: 227-231.

25. Carroll, B. Letters to the editor. J Clin Ultrasound 1981, 9: A30-A31.

26. Strijk, S.P., Boetes, C. \& Rosenbusch, G. Floating stones in a nonopacified gallbladder: ultrasonographic sign of gas containing gallstones. Gastrointest Radiol 1979, 133: 435-436.

27. Becker, C.D. \& Vock, P. Appearance of gas-containing gallstones on sonography and computed tomography. Gastrointest Radiol 1984, 9: 323-328.

28. Lebensart, P.D., Bloom R.A., Meretyk, S., Landau, E.H. \& Shiloni, E. Oral cholecystosonography: a method for facilitating the diagnosis of cholesterol gallstones. Radiology 1984, 153: 255-256.

29. Shlaer, W.J., Leopold, G.R. \& Scheible, F.W. Sonography of the thickened gallbladder wall: a non-specific finding. AJR 1981, 136: 337-339.

30. Shuman, W.P., Rogers, J.V., Rudd, T.G., Mack, L.A., Plumley, T. \& Larson, E.B. Low sensitivity of sonography and cholescintigraphy in acalculous cholecystitis. AJR 1984, 142. 531-534.

31. Filly, R.A., Allen, B., Minton, M.J., Bernhoft, R., Way, L.W. In vitro investigation of the origin of echoes within biliary sludge. J Clin Ultrasound 1980, 8: 193200.

32. Anastasi, B. \& Sutherland, G.R. Biliary sludge ultrasonic appearance simulating neoplasm. Br J Radiol 1981, 54: 679-681.

33. MacDonald, F.P., Cooperberg, P.L. \& Cohen, M.M. The WES triad: a specific sonographic sign of gallstones in the contracted gallbladder. Gastrointest Radiol 1981, 6: $39-41$.

34. Raptopoulos, V.D., D'Orsi, C., Smith, E.H., Reuter, K., Moss, L. \& Kleinman, P. Dynamic cholecystosonography of the contracted gallbladder: the double-arcshadow sign. AJR 1982, 138: 275-278.

35. Garmendia, F.S., Ruiz, J.A.L., Alvarez, A.M., Siınago, J.M.P., Ratia, J.A.C. \& Dermit, F.M. Bouveret's syndrome: new cause of double-arc-shadow sign in cholecystosonography. Eur J Radiol 1984, 9: 346-347.

36. Yem, H.C. Update on the gallbladder. In Saunders, R.C. (ed) Ultrasound Annual. Raven Press, New York, 1982, pp. 23-25.

37. Kane, R.A., Jacobs, R., Katz, J. \& Costello, P. Porcelain gallbladder: ultrasound and CT appearance. Radiology 1984, 152: 137-141.

38. Graif, M., Horovitz, A., Itzchak, Y. \& Strauss, S. Hyperechoic foci in the gallbladder wall as a sign of 
microabscess formation of diverticular. Radiology 1984, 152: $781-784$.

39. Polk, H.C. Carcinoma and the calcified gallbladder. Gastroenterology 1966, 50: 582-585.

40. Blaquiere, R.M. \& Dewbury, K.C. The ultrasound diagnosis of emphysematous cholecystitis. Br J Radiol 1981, 55: $114-116$.

41. Bloom, R.A., Fisher, A., Pode, D. \& Asaf, Y. Shifting intramural gas: a new ultrasound sign of emphysematous cholecystitis. J Clin Ultrasound 1984, 12: $40-42$.
42. Raptopoulos, V.D. Ultrasonic pseudocalculus effect in postcholecystectomy patients. $A J R$ 1980, 134: 145-148.

43. Lewandowski, B., French, G. \& Winsberg, F. Normal postcholecystectomy sonogram: gas vs. clips. $J \subseteq$ Ultrasound Med 1985, 4: 7-12.

44. Way, L.W., Sleisenger, M.H. Cholelithiasis and chronic $\stackrel{\vec{F}}{\stackrel{\oplus}{ }}$ cholecystitis. In Sleisenger, M.H. \& Fordtran, J.S. (ed): Gastrointestinal Disease, 2nd edition. W.B. Saunders, Philadelphia, 1978, 1481-1486.

45. Laing, F.C. Diagnostic evaluation of patients with susp- $\mathbb{\Phi}$ ected cholecystitis. Surg Clin North Am 1984, 64: 3-22. 